\title{
УДК 622.32:519.8
}

\section{КОМПЛЕКСНЫЙ ПОДХОД К ПАРАМЕТРИЗАЦИИ ГЕОЛОГО-ГИДРОДИНАМИЧЕСКОЙ МОДЕЛИ ДЛЯ ЕЁ АВТОАДАПТАЦИИ К ПРОЦЕССАМ РАЗРАБОТКИ ЗАЛЕЖЕЙ НЕФТИ}

\author{
Еремян Грачик Араикович1', \\ eremyanga@hw.tpu.ru
}

\author{
Давуди Шадфар 1 , \\ davoodis@hw.tpu.ru
}

Рукавишников Валерий Сергеевич1,
rukavishnikovvs@hw.tpu.ru

Степико Артем Вадимович1, stepikoav@hw.tpu.ru 1 Национальный исследовательский Томский политехнический университет,
Россия, 634050, г. Томск, пр. Ленина, 30.

\begin{abstract}
Актуальность исследования обусловлена необходимостью выбора из множества параметров геолого-гидродинамической модели месторождения углеводородов именно тех, за счет настройки которых алгоритм оптимизации будет находить решения, обеспечивающие корректное воспроизведение исторических данных моделью. Адаптация модели на историю эксплуатации залежи углеводородов является трудоемким и неотъемлемым этапом численного моделирования резервуаров углеводородов, подтверждающим пригодность модели для прогнозов. Ограничение числа настроечных параметров адаптации до наиболее значимых дает возможность модели быть успешно адаптированной на практике в рамках разумных временных заmpam.

Цель: определить набор значимых параметров адаптации модели на основе изучения степени и характера их влияния на сходимость ключевых показателей работы скважин и месторождения.

Объект: автоматизированная адаптация синтетической модели нефртяного месторождения на данные эталонного расчета, являющегося аналогом исторических показателей разработки месторождения.

Методы: геолого-гидродинамическое моделирование, вычислительный эксперимент, анализ чувствительности.

Результаты. Составлена классификация параметров адаптации модели по степени влияния и степени неопределенности исходя из закона течения флюида в пористой среде. На основе множественных вычислительных экспериментов на построенной в ходе работы синтетической геолого-гидродинамической модели изучена степень и характер влияние различных параметров адаптации на сходимость показателей разработки месторождения. Применение двух видов анализа чувствительности позволило охарактеризовать как отдельное влияние параметров, так и комбинированное влияние с учетом варьирования остальных параметров. Понимание взаимовлияния параметров важно, поскольку при адаптации модели варьируются сразу все настроечные параметры. Выводы численных экспериментов согласуются с аналитическим подходом. Реализованный комплексный подход позволяет сформировать обоснованный набор настроечных параметров модели, ранжированный по степени важности для адаптации.
\end{abstract}

\section{Ключевые слова:}

Анализ чувствительности, параметризация модели, параметры адаптации, неопеделенности, автоматизированная адаптация, алгоритм оптимизации, целевая функция, геолого-гидродинамическое моделирование.

\section{Введение}

Компьютерное моделирование разработки месторождений углеводородов используется в качестве вспомогательного инструмента для наиболее полного извлечения запасов сырья при максимальной экономической рентабельности. Для подтверждения пригодности модели для прогнозов сначала необходимо произвести процедуру адаптации или «калибровки» параметров модели таким образом, чтобы она корректно воспроизводила историю разработки месторождения. Основанием для адаптации параметров, заложенных в модель, являются присущие им диапазоны неопределенности. Адаптация модели является классической обратной задачей [1].

Типичным подходом к задаче адаптации гидродинамических моделей является ручная настройка параметров модели, содержащих в себе наибольшую неопределенность [2]. Данному подходу присущ ряд недостатков, в числе которых большие временные затраты, единственное решение, недооценка неопределенностей в данных и прогнозе. В качестве альтернативного подхода используются алгоритмы автоматизированной адаптации, включая машинное обучение и нейронные сети. Подходы и алгоритмы автоматизированной адаптации описаны в ряде работ [3-15]. В той или иной степени методы автоадаптации позволяют улучшить надежность и эффективность применения гидродинамических моделей. Суть автоматизированной адаптации модели заключается в минимизации целевой функции, которая представляет собой меру расхождения между модельным расчетом и историей добычи и является критерием качества адаптации. Минимизация целевой функции производится с помощью математических оптимизационных алгоритмов, которые итеративно подбирают значение параметров модели в рамках их диапазонов неопре- 
деленности. Более подробно роль, виды и эффективность целевых функций применительно к задаче автоадаптации описаны в работах [16-23].

До начала процесса адаптации модели необходимо выполнить параметризацию, то есть из множества параметров модели выбрать именно те, за счет настройки которых оптимизационный алгоритм будет находить решения, обеспечивающие корректное воспроизведение исторических данных. Если бы вычислительные мощности не были бы ограничены, то для оптимизации можно было бы задать весь набор всевозможных параметров адаптации, в истинных значениях которых существует некоторая неопределенность, а затем найти решения с помощью простого стохастического перебора комбинаций (метод Монте Карло). Поскольку описанный выше пример носит чисто гипотетический характер, инженеру по моделированию разработки реального месторождения необходимо выбрать те параметры, которые обладают наибольшей неопределенностью и в то же время существенно влияют на динамику течения флюида в пласте. Ограничение числа настроечных параметров адаптации до наиболее значимых дает возможность модели быть успешно адаптированной на практике в рамках разумных временных затрат. Таким образом, выявление, оценка и оцифровка неопределенностей являются важными этапами в построении геологогидродинамической модели [24, 25]. Примеры подходов к количественной оценке неопределенностей детально описаны в работах [26-29].

Целью данной работы является определение набора значимых параметров адаптации модели на основе комплексного изучения степени и характера их влияния на сходимость ключевых показателей разработки месторождения.

Новизна данной работы заключается в комплексировании четырех подходов к параметризации модели: на основе анализа уравнения фильтрации, простого и расширенного анализов чувствительности, на основе численных экспериментов, а также на основе визуального анализа сходимости графиков показателей работы скважины.

\section{Подходы и методы исследования}

Для решения задачи параметризации возможно применение двух основных подходов. Первый подход основан на теоретическом анализе взаимосвязи параметров модели с законом течения флюида в пористой среде, также известным как закон Дарси [30].

Второй подход к выбору параметров адаптации основан на анализе чувствительности результатов расчета модели (например, накопленной добычи нефти) к изменению значений параметров адаптации [31-37]. При данном методе сначала выявляются и оцифровываются всевозможные геологические, петрофизические и прочие неопределенности параметров модели. Далее проводится расчет цифровой модели месторождения при поочередном варьировании значений параметров адаптации в пределах заданных диапазонов неопределенностей. По результатам расчетов строятся торнадо-диаграммы, на которых рас- полагают параметры в порядке от наиболее влияющего к наименее влияющему, таким образом давая визуальное представление о степени влияния параметров. Некоторые коммерческие программы для геологогидродинамического моделирования позволяют на основе результатов расчета моделей для анализа чувствительности построить торнадо-диаграмму для продвинутого анализа чувствительности. Отличие продвинутой модификации в том, что она позволяет увидеть комбинированное влияние параметров адаптации на основе прокси-моделирования и расчета коэффициентов корреляции. Понимание взаимовлияния параметров важно, поскольку при адаптации модели варьируются сразу все настроечные параметры. Дополнительно в комплексный подход к параметризации модели включается визуальный анализ сходимости показателей работы скважин по графикам.

Для выполнения анализа чувствительности, основанного на серии вычислительных экспериментов, в рамках работы построена синтетическая геологогидродинамическая модель (рис. 1).

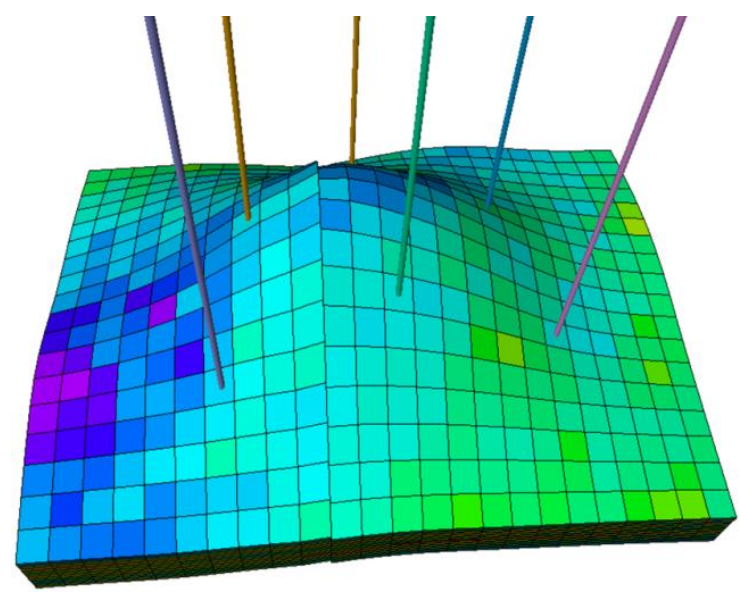

Puc. 1. Синтетическая 3D модель нефтяной залежи

Fig. 1. Synthetic 3D model of an oil reservoir

Модель представляет собой типичную пластовосводовую нефтяную залежь. В концепцию модели заложена прибрежно-морская обстановка осадконакопления по аналогии с одним из месторождений Томской области. Толщина песчаного коллектора варьируется в диапазоне от 11 до 14 м, средняя проницаемость равна 50 мД. Модель состоит из 6800 ячеек и включает в себя 6 скважин, 3 из которых добывают нефть, остальные 3 закачивают воду в пласт. Таким образом имитируется разработка залежи методом заводнения. Для того чтобы эксперименты по адаптации были возможны, необходимо было создать аналогию исторических показателей работы скважин. Модель рассчитывается в режиме прогноза при заданных забойных давлениях на всех скважинах и при исходных («истинных») значениях параметров модели. Далее получившиеся показатели работы скважин, такие как дебиты, приемистости и давления, выгружаются в качестве данных истории, характеризуя эталонную модель. В данном случае создана история для 7 лет работы скважин. 
Синтетическая модель существенно меньше и проще моделей большинства реальных месторождений. В то же время она состоит из тех же элементов и подчиняется тем же законам фильтрации флюида в пористой среде, как и модели реальных месторождений углеводородов.

\section{Результаты}

Уравнение дебита скважины для установившегося режима течения описывается формулой (1), которая является радиальной формой закона Дарси и получила название формулы Дюпюи [38].

$$
Q=\frac{2 \pi k h}{B \mu\left(\ln \frac{r_{e}}{r_{w}}-\frac{1}{2}+S_{\text {обш }}\right)}\left(\overline{P_{\text {пл }}}-P_{\text {заб }}\right),
$$

где $Q$ - дебит флюида; $\overline{P_{\text {пл }}}$ - среднее пластовое давление; $P_{\text {заб }}$ - забойное давление; $k$ - эффективная проницаемость по флюиду; $h$ - толщина коллектора; $B$ объемный коэффициент флюида; $\mu$ - коэффициент вязкости флюида; $r_{e}$ - радиус контура питания; $r_{w}-$

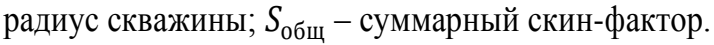

Правая часть формулы (1) является произведением депрессии, представленной разностью пластового и забойного давления, и коэффициента продуктивности, представленного остальной частью произведения (формула (2)).

$$
P I=\frac{2 \pi k h}{B \mu\left(\ln \frac{r_{e}}{r_{w}}-\frac{1}{2}+S_{\text {общ }}\right)},
$$

где $P I$ - коэффициент продуктивности.

Таким образом, коэффициент продуктивности скважины может быть выражен как дебит, деленный на депрессию (формула (3)), показывая какой суточный объем флюида добывается скважиной за счет единицы перепада давления. Физически коэффициент продуктивности скважины характеризует фильтрационные свойства пласта и призабойной зоны скважины.

$$
\frac{2 \pi k h}{B \mu\left(\ln \frac{r_{e}}{r_{w}}-\frac{1}{2}+S_{\text {обш }}\right)}=\frac{Q}{\left(\overline{P_{\text {пл }}}-P_{\text {заб }}\right)} .
$$

Правая часть формулы (3) описывается историческими данными разработки скважин и месторождения в целом. Параметры модели, входящие в левую часть и/или влияющие на нее, обладают некоторой степенью неопределенности, поэтому требуется подобрать их значения в процессе оптимизации таким образом, чтобы результаты расчета модели соответствовали историческим данным.

В табл. 1 представлены параметры адаптации, условно разделенные на 6 групп на основании природы их влияния на продуктивность скважины. Показаны наиболее влияющие параметры адаптации для разбуренного месторождения исходя из закона течения флюида в пористой среде.

Чем сильнее параметр влияет на коэффициент продуктивности, тем сильнее его влияние на сходимость расчетных дебитов и давлений с историей, то есть на адаптацию. Параметры, оказывающие сильное влияние на адаптацию и в то же время обладающие наибольшей неопределенностью, как правило, связаны с распределением эффективной проницаемости в пространстве и во времени. Эффективной проницаемостью называется проницаемость, характеризующая течение данной фазы при наличии в коллекторе других флюидов. Как правило, модель калибруется варьированием коэффициентов абсолютной проницаемости, относительных фазовых проницаемостей (ОФП), вертикальной анизотропии проницаемости, проводимости разломов и тому подобного. Наглядные примеры описаны в работах $[39,40]$.

Таблица 1. Наиболее влияющие параметры адаптации для разбуренного месторождения исходя из закона течения флюида в пористой среде

Table 1. The most influencing history matching

\begin{tabular}{|c|c|c|c|}
\hline $\begin{array}{l}\text { Группа } \\
\text { Group }\end{array}$ & $\begin{array}{c}\text { Параметры адаптации } \\
\text { History matching } \\
\text { parameters }\end{array}$ & $\begin{array}{c}\text { Степень } \\
\text { влияния } \\
\text { Influence }\end{array}$ & $\begin{array}{l}\text { Степень } \\
\text { неопр. } \\
\text { Uncertainty }\end{array}$ \\
\hline 1 & $\begin{array}{l}\text { Проницаемость (абсо- } \\
\text { лютная, эффективная) } \\
\text { Permeability (absolute, } \\
\text { effective) }\end{array}$ & $\begin{array}{l}\text { Сильное } \\
\text { Strong }\end{array}$ & $\begin{array}{l}\text { Высокая } \\
\text { High }\end{array}$ \\
\hline 2 & $\begin{array}{l}\text { Параметры, влияющие } \\
\text { на проницаемость (пори- } \\
\text { стость, водонасыщен- } \\
\text { ность и прочие) } \\
\text { Parameters affecting } \\
\text { permeability } \\
\text { (porosity, water saturation } \\
\text { and others) }\end{array}$ & $\begin{array}{l}\text { Умеренное } \\
\text { Moderate }\end{array}$ & $\begin{array}{l}\text { Средняя } \\
\text { Medium }\end{array}$ \\
\hline 3 & $\begin{array}{l}\text { Толщина коллектора } \\
\text { Reservoir thickness }\end{array}$ & $\begin{array}{l}\text { Сильное } \\
\text { Strong } \\
\end{array}$ & $\begin{array}{l}\text { Низкая } \\
\text { Low }\end{array}$ \\
\hline 4 & $\begin{array}{l}\text { Вязкость и объемный } \\
\text { коэффициент } \\
\text { Viscosity and formation } \\
\text { volume factor }\end{array}$ & $\begin{array}{l}\text { Сильное } \\
\text { Strong }\end{array}$ & $\begin{array}{l}\text { Низкая } \\
\text { Low }\end{array}$ \\
\hline 5 & $\begin{array}{l}\text { Скин-фактор } \\
\text { Skin factor }\end{array}$ & $\begin{array}{l}\text { Умеренное } \\
\text { Moderate }\end{array}$ & $\begin{array}{l}\text { Средняя } \\
\text { Medium }\end{array}$ \\
\hline 6 & $\begin{array}{l}\text { Геометрия скважины и } \\
\text { зоны дренирования } \\
\text { Well geometry and } \\
\text { drainage area }\end{array}$ & $\begin{array}{l}\text { Умеренное } \\
\text { Moderate }\end{array}$ & $\begin{array}{l}\text { Низкая } \\
\text { Low }\end{array}$ \\
\hline
\end{tabular}
parameters for a developed field based on the law of fluid flow in a porous medium

После теоретического анализа параметризации модели в данной работе проводится серия вычислительных экспериментов на построенной синтетической модели. Каждый настроечный параметр модели, теоретически способный существенно влиять на расчет модели, был протестирован численным экспериментом, чтобы убедиться в степени и характере влияния тестируемого параметра. Список выбранных параметров адаптации с указанием их буквенных обозначений, истинных значений и диапазонов варьирования приведен в табл. 2.

В качестве параметров адаптации выбрано 8 параметров, отвечающих за проницаемость, водонасыщенность и уровень зеркала свободной воды. В качестве уравнения взаимосвязи проницаемости от пористости используется формула вида

$$
\ln k=a \varphi+b,
$$


где $k$ - проницаемость; $\varphi$ - пористость; $a$ и $b$ - коэффициенты уравнения.

Анализ чувствительности накопленных показателей разработки залежи к изменению параметров адаптации позволяет ранжировать и упорядочивать параметры по степени влияния (рис. 2).

Расчет моделей при адаптации производится с контролем расходов жидкости, что означает, что количество добытой и закаченной жидкости в модели должно строго соответствовать тем же показателям по истории работы скважин. Диаграмма Торнадо для накопленной добычи жидкости свидетельствует о том, что варьирование коэффициентов Corey_water, Perm_a и Perm_b может привести к недобору жидкости скважинами модели, что, несомненно, приведет к нарушению материального баланса. Перечисленные коэффициенты сильно влияют на эффективную про- ницаемость, входящую в уравнение фильтрации флюида в пористой среде. Данный эффект происходит при недостатке продуктивности пласта даже при использовании максимального перепада давления.

По степени влияния на накопленную добычу нефти распределение настроечных параметров выглядит иначе. Наиболее влияющим параметром является FWL, поскольку он определяет вскрытую нефтенасыщенную толщину. Его варьирование может отражаться на накопленной добыче нефти в диапазоне от $39 \%$ недобора до $42 \%$ перебора. Ожидаемо сильно влияют коэффициенты уравнения ОФП и уравнения пористость-проницаемость. Влияние вертикальной анизотропии Kv_Kh и водонасыщенности в переходной зоне $\mathrm{Sw}$ _а более слабое, поскольку добывающие скважины вертикальные и пробурены в чистую нефтяную зону.

Таблица 2. Параметры адаптации модели

Table 2. History matching parameters of the model

\begin{tabular}{|c|c|c|c|c|c|}
\hline № & $\begin{array}{c}\text { Параметры адаптации } \\
\text { History matching parameters }\end{array}$ & $\begin{array}{l}\text { Обозначение } \\
\text { Designation }\end{array}$ & $\begin{array}{l}\text { Мин } \\
\text { Min }\end{array}$ & $\begin{array}{l}\text { Макс } \\
\text { Мax }\end{array}$ & $\begin{array}{l}\text { Истина } \\
\text { Truth }\end{array}$ \\
\hline 1 & $\begin{array}{l}\text { Глубина зеркала свободной воды, м } \\
\text { Free water level, m }\end{array}$ & \$FWL & -2455 & -2445 & -2450 \\
\hline 2 & $\begin{array}{l}\text { Степень уравнения Кори по нефти } \\
\text { Oil exponent in Corey equation }\end{array}$ & \$Corey_O_W & 3,0 & 8,0 & 5,0 \\
\hline 3 & $\begin{array}{l}\text { Степень уравнения Кори по воде } \\
\text { Water exponent in Corey equation }\end{array}$ & \$Corey_water & 0,6 & 5,0 & 1,2 \\
\hline 4 & $\begin{array}{l}\text { Концевая точка ОФП по воде } \\
\text { End point of water relative permeability }\end{array}$ & \$Krw_Sorw & 0,3 & 0,9 & 0,7 \\
\hline 5 & $\begin{array}{l}\text { Коэффициент «а» в уравнении проницаемости от пористости } \\
\text { Coefficient «a» in poro-perm equation }\end{array}$ & \$Perm_a & 64 & 74 & 70 \\
\hline 6 & $\begin{array}{l}\text { Коэффициент «b» в уравнении проницаемости от пористости } \\
\text { Coefficient «b» in poro-perm equation }\end{array}$ & \$Perm_b & 8,0 & 9,0 & 8,2 \\
\hline 7 & $\begin{array}{l}\text { Вертикальная анизотропия проницаемости, д.е. } \\
\text { Vertical anisotropy of permeability, unit fraction }\end{array}$ & \$Kv_Kh & 0,01 & 0,30 & 0,20 \\
\hline 8 & $\begin{array}{l}\text { Коэффициент уравнения Ј-функции } \\
\text { Coefficient in J-function }\end{array}$ & \$Sw_a & $-0,20$ & $-0,14$ & $-0,18$ \\
\hline
\end{tabular}
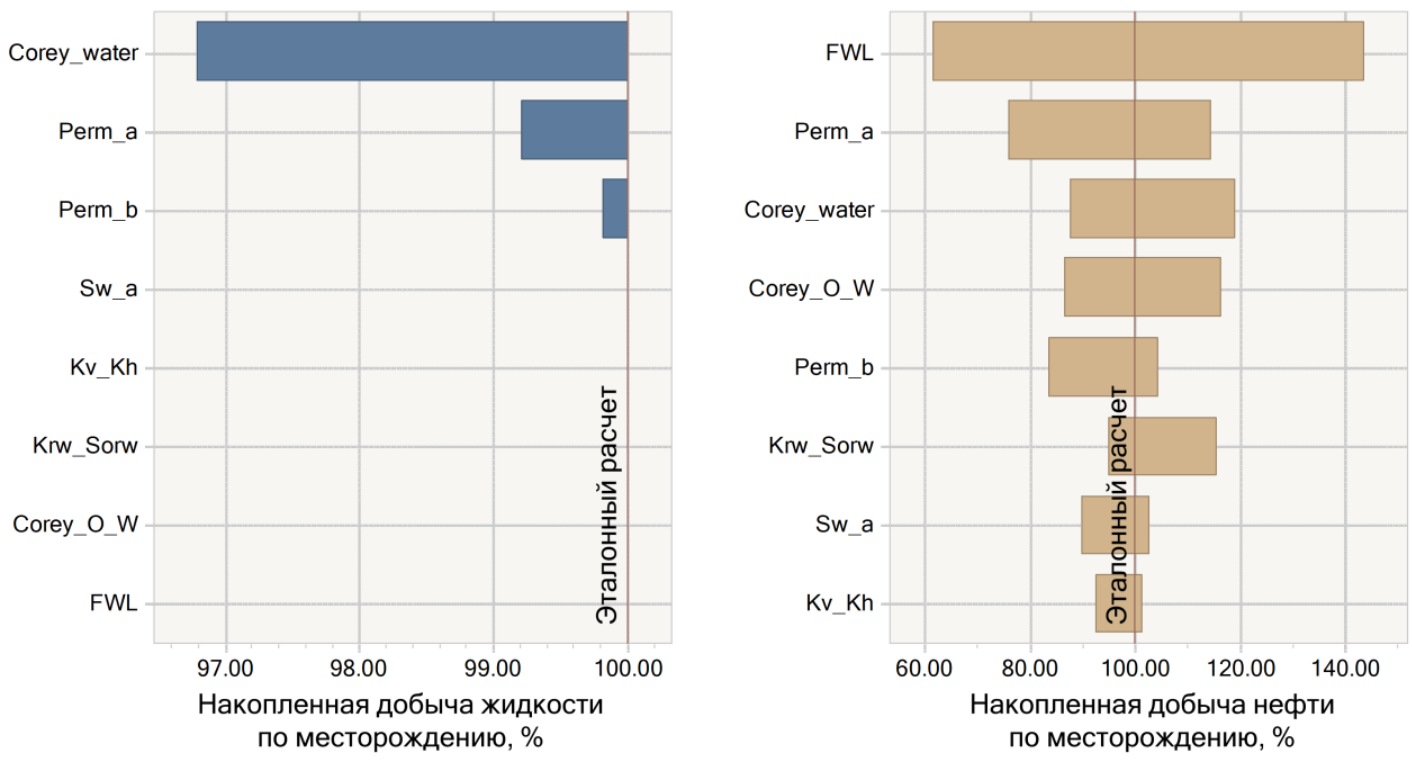

Pис. 2. Диаграммы Торнадо на основе анализа чувствительности

Fig. 2. Tornado charts based on sensitivity analysis

Более наглядным с инженерной точки зрения является анализ графиков показателей работы скважин: дебитов жидкости, нефти, воды и забойных давлений. На примере скважины А видно, в каких широких диапазонах изменяется сходимость показателей при варьировании настроечного параметра Corey_Water (рис. 3). 

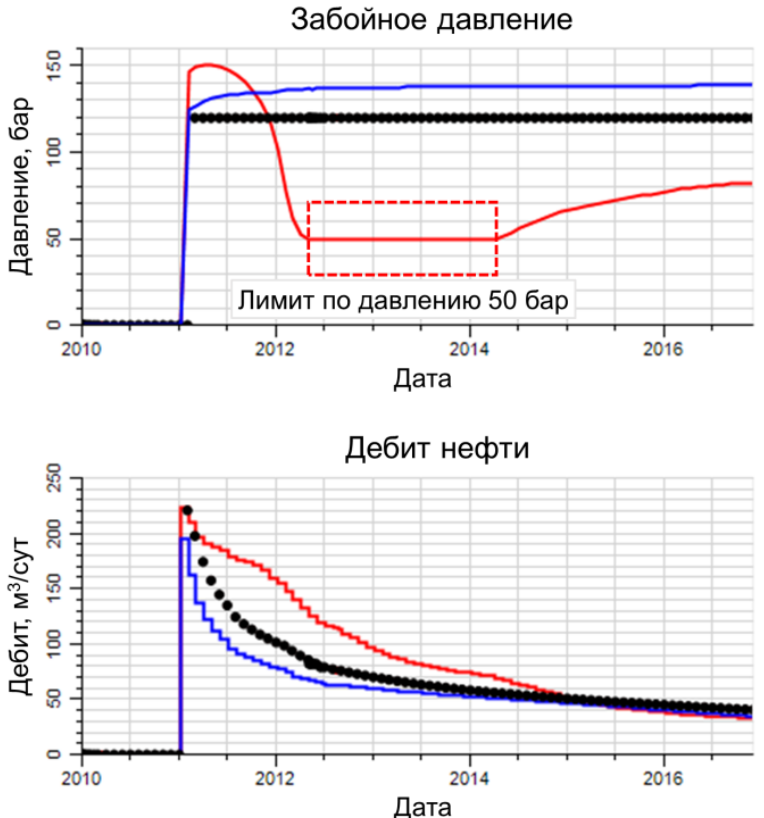

_— Расчет при минимальном значении Corey_Water

—_ Расчет при максимальном значении Corey_Water

Pис. 3. Показатели работы скважины А при минимальном и максимальном значениях параметра Соrеy_Water

Fig. 3. Performance indicators of well A at minimum and maximum values of the parameter Corey_Water

Участок недобора жидкости возникает в том же временном периоде, где забойное давление принимает минимальное значение 50 бар, ограниченное технологическим режимом работы скважины. Причиной является сильное расхождение пропорции добываемых флюидов: мало воды и недостаточно много нефти для компенсации недобора жидкости. Из этого следует, что диапазон неопределенности настроечно-
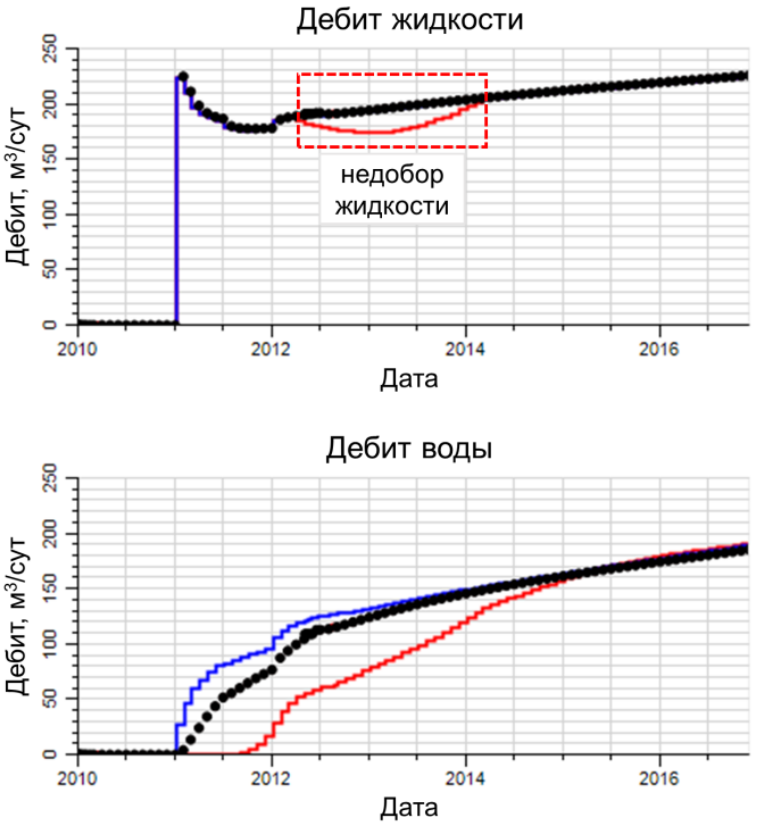

Исторические данные

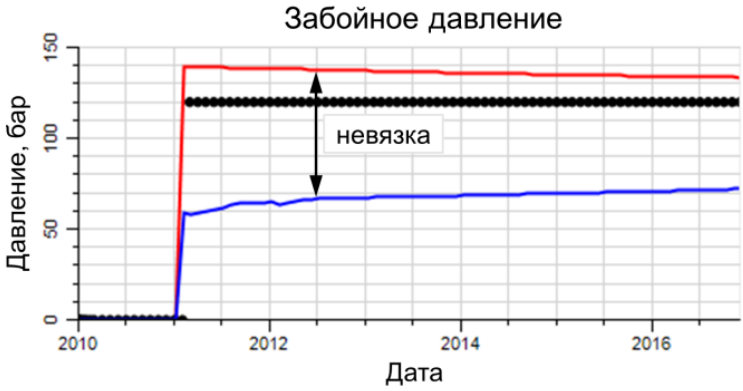

го параметра Corey_Water может быть сужен для того, чтобы избежать нарушения материального баланса и сэкономить вычислительные мощности на расчете заведомо неудовлетворительных вариантов модели.

На примере графиков работы той же скважины при варьировании параметра Perm_a наблюдается различие в характере влияния параметра на сходимость показателей (рис. 4).
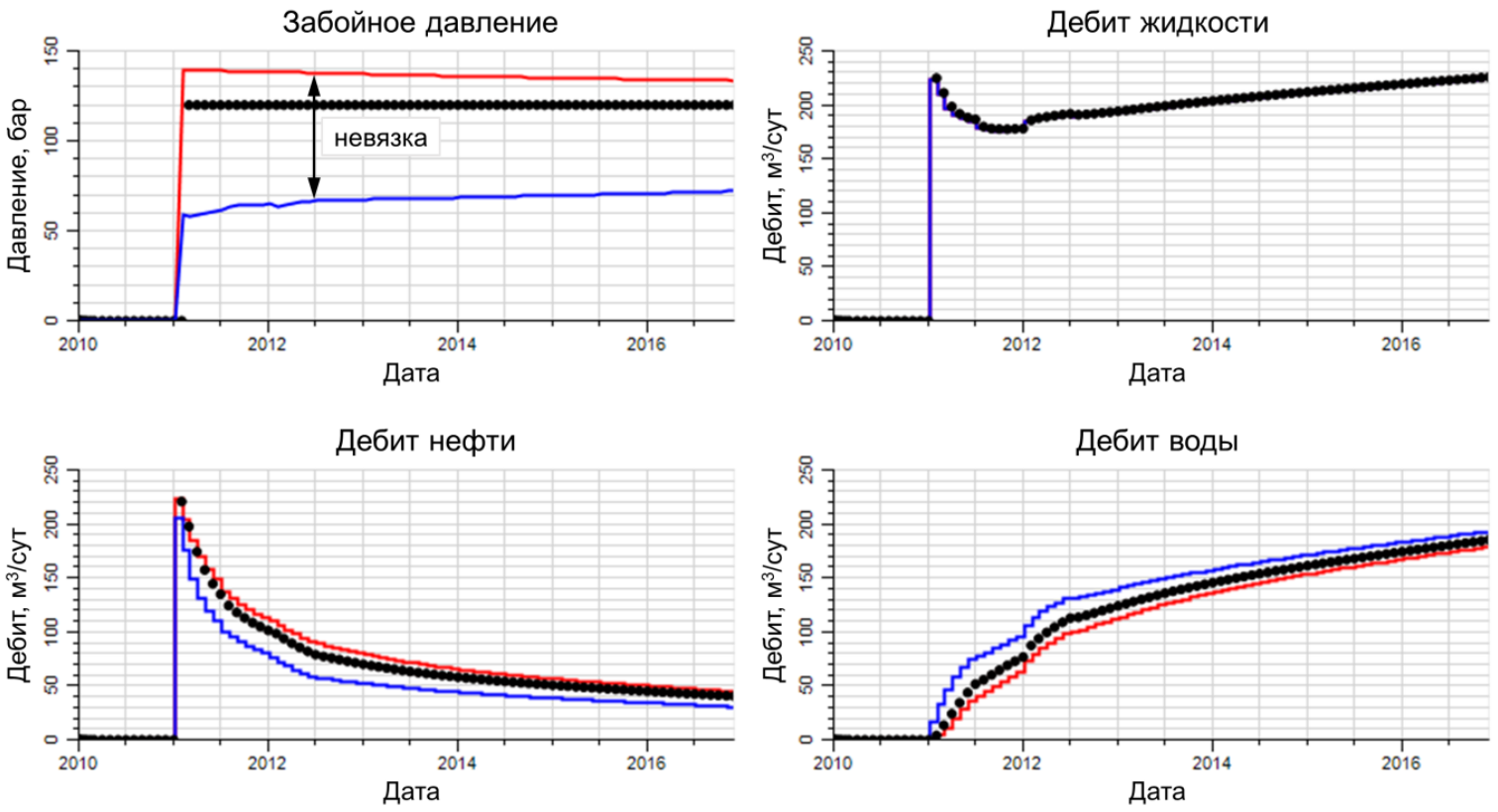

—— Расчет при минимальном значении Perm_a

Исторические данные

—— Расчет при максимальном значении Perm_a

Рис. 4. Показатели работы скважины А при минимальном и максимальном значениях параметра Регт_a

Fig. 4. Performance indicators of well A at minimum and maximum values of the parameter Perm_a 
Поскольку от параметра Perm_a прямо пропорционально зависит продуктивность скважины, это отражается на широком диапазоне расхождения забойного давления. Влияние на соотношение дебитов воды и нефти существенно слабее.

В целевую функцию в данной работе включены: дебит нефти, дебит воды, приемистость воды, забойные и пластовые давления по всем 6 скважинам в соответствии с методикой выбора оптимального вида целевой функции [41]. При адаптации модели ориентиром для алгоритма оптимизации является значение целевой функции. В связи с этим имеет смысл по- строить диаграмму Торнадо для анализа чувствительности целевой функции к параметрам адаптации. В дополнение к простому анализу чувствительности в используемом программном комплексе для каждого параметра адаптации на основе проксимоделирования рассчитаны весовые коэффициенты, учитывающие комбинированное влияние данного параметра при одновременном варьировании других параметров. Полученные веса позволяют на ряду с простой диаграммой Торнадо построить подобную диаграмму для расширенного анализа чувствительности (рис. 5).
Простой анализ чувствительности

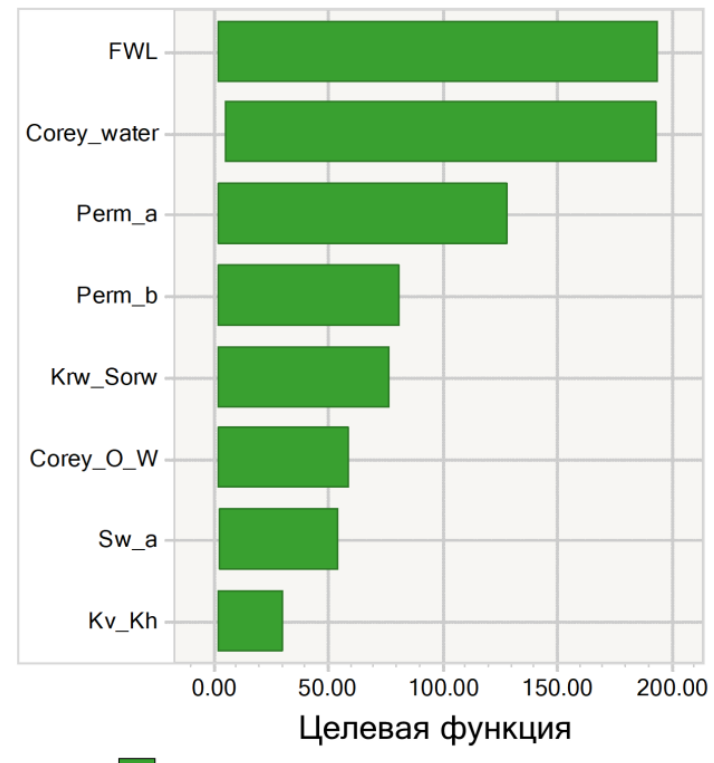

Индивидуальный вклад
Расширенный анализ чувствительности

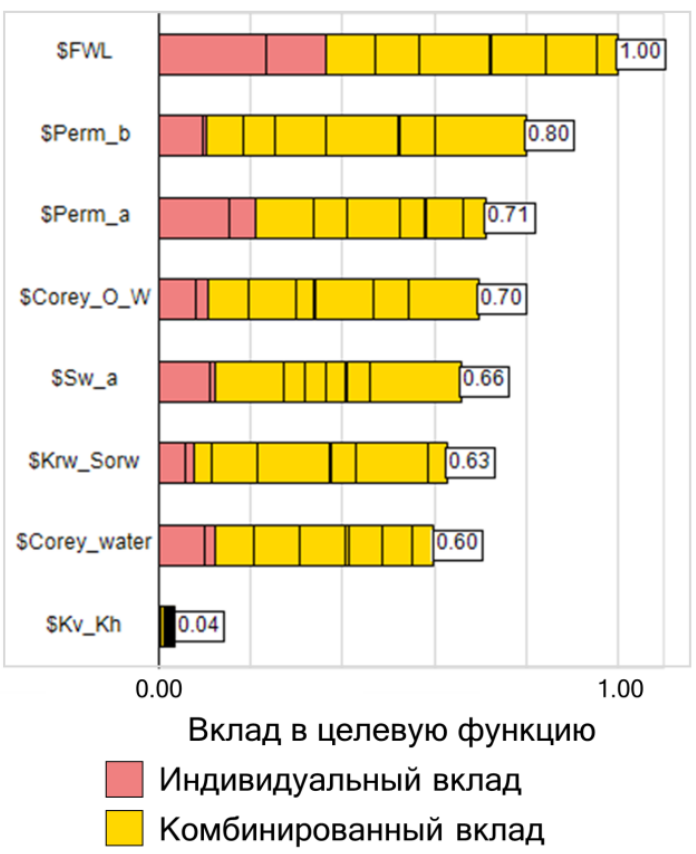

Рис. 5. Сравнение результатов простого и расширенного анализов чувствительности

Fig. 5. Comparison of the results of simple and advanced sensitivity analyzes

Простой анализ чувствительности говорит о том, что можно выделить три самых влияющих параметра из восьми: FWL, Corey_water и Perm_a. Однако расширенный анализ чувствительности показывает, что все используемые параметры, кроме $\mathrm{Kv}_{-} \mathrm{Kh}$, сильно влияют на значение целевой функции и, соответственно, на адаптацию. Таким образом, получаем новый и более уверенный вывод о том, что для задачи адаптации целесообразно отказаться только от параметра вертикальной анизотропии проницаемости $\mathrm{Kv} \_\mathrm{Kh}$, остальные следует использовать.

После применения обозначенных подходов к параметризации следует произвести комплексирование результатов. Теоретический анализ на основе уравнения фильтрации помог выделить общие группы параметров модели по степени влияния и степени неопределенности. Анализ чувствительности на основе численных экспериментов позволил отранжировать 8 выбранных параметров по степени влияния на адаптацию конкретной модели нефтяной залежи. Благодаря расширенному анализу чувствительности удалось сделать вывод, что параметр $\mathrm{Kv} \_\mathrm{Kh}$ можно исклю- чить без ущерба для адаптации модели. Анализ графиков показателей работы скважин позволяет увидеть характер влияния каждого параметра, что ценно с инженерной точки зрения.

\section{Выводы}

Комплексный подход к параметризации модели нефтяной залежи для автоадаптации позволяет визуализировать и понять степень и характер влияния конкретных параметров адаптации на сходимость показателей работы скважин и месторождения.

Применение двух видов анализа чувствительности позволило охарактеризовать как отдельное влияние параметров, так и влияние с учетом варьирования остальных параметров. Понимание взаимовлияния параметров важно, поскольку при адаптации модели варьируются сразу все настроечные параметры. Выводы численных экспериментов согласуются с описанным аналитическим подходом.

Реализованный комплексный подход позволил сформировать обоснованный набор настроечных параметров для созданной геолого-гидродинамической 
модели нефтяной залежи, ранжированный по степени важности для автоадаптации процесса её разработки. Ограничение числа настроечных параметров адаптации до наиболее значимых сокращает вычислительные затраты алгоритма оптимизации благодаря

\section{СПИСОК ЛИТЕРАТУРЫ}

1. Tarantola A. Inverse problem theory and methods for model parameter estimation. - Paris: Society for Industrial and Applied Mathematics, 2005. $-342 \mathrm{p}$.

2. Гладков Е.А., Гладкова Е.Е. Трехмерная геологотехнологическая модель месторождения УВ на основе индивидуальной поскважинной адаптации // Газовая промышленность. - 2010. - № 5. - С. 36-39.

3. Геологически обоснованная автоматизированная адаптация гидродинамических моделей на примере реального месторождения / Г.Ю. Шишаев, И.В. Матвеев, Г.А. Еремян, В.В. Демьянов, С.В. Кайгородов // Нефтяное хозяйство. - 2020. - № 6. C. 58-61.

4. Geology driven history matching / I. Matveev, G. Shishaev, G. Eremyan, V. Demyanov， O. Popova， S. Kaygorodov, B. Belozerov, J. Uzhegova, D. Konoshonkin, M. Korovin // SPE Russian Petroleum Technology Conference. - Moscow, 22-24 October, 2019. URL: https://onepetro.org/SPERPTC/proceedingsabstract/ 19RPTC/ 1-19RPTC/D013S001R004/219147 (дата обрашения: 05.04.2021).

5. Geology realism control in automated history matching / I. Matveev, G. Shishaev, G. Eremyan, D. Konoshonkin, V. Demyanov, S. Kaygorodov // ECMOR XVII: Conference Proceedings. - Houten, 14-17 September, 2020. - Houten: European Association of Geoscientists \& Engineers Publ., 2020. V. 2020. - P. 1-9.

6. Verga F., Cancelliere M., Viberti D. Improved application of assisted history matching techniques // Journal of Petroleum Science and Engineering. - 2013. - V. 109. - P. 327-347.

7. Foroud T., Seifi A., Amin Shahidi B. Assisted history matching using artificial neural network based global optimization method Applications to Brugge field and a fractured Iranian reservoir // Journal of Petroleum Science and Engineering. - 2014. - V. 123. P. 46-61.

8. An integrated closed-loop solution to assisted history matching and field optimization with machine learning techniques / Z. Chai A. Nwachukwu, Y. Zagayevskiy, S. Amini, S. Madasu // Journal of Petroleum Science and Engineering. - 2021. - V. 198. - 108204

9. Genetic Algorithm for the History Matching Problem / C.R. Xavier, E.P. Santos, V.F. Vieira, R.W. Santos // Procedia Computer Science. - 2013. - V.18. - P. 946-955.

10. Rwechungura R.W., Dadashpour M., Kleppe J. Advanced history matching techniques reviewed // SPE Middle East Oil and Gas Show and Conference. - Manama, Bahrain, September, 2011. URL: https://onepetro.org/SPEMEOS/proceedings-abstract/11MEOS/ All-11MEOS/SPE-142497-MS/148210 (дата обращения: 05.04.2021).

11. Almeida F.L.R., Davolio A., Schiozer D.J. A New approach to perform a probabilistic and multi-objective history matching // SPE Annual Technical Conference and Exhibition. - Amsterdam, Netherlands, October, 2014. URL: https://onepetro.org/ SPEATCE/proceedings-abstract/14ATCE/All-14ATCE/SPE170623-MS/211614 (дата обращения: 05.04.2021).

12. Al-Sofi A.M., Blunt M.J. Control of numerical dispersion in simulations of augmented waterflooding // SPE Improved Oil Recovery Symposium. - Tulsa, USA, April 2010. URL: https://onepetro.org/ SPEIOR/proceedings-abstract/10IOR/All10IOR/SPE-129658-MS/107593 (дата обрашения: 05.04.2021).

13. Joint history matching of production and 4D-seismic related data for a North Sea field case / V. Gervais-Couplet, F. Roggero, M. Feraille, M. Le Ravalec-Dupin, A. Seiler // SPE Annual Technical Conference and Exhibition. - Florence, Italy, September 2010. URL: https://onepetro.org/SPEATCE/proceedingsabstract/10ATCE/ All-10ATCE/SPE-135116-MS/102106 (дата обращения: 05.04.2021)

14. Kazemi A., Stephen K.D. Optimal parameter updating in assisted history matching using streamlines as a guide // Oil \& Gas Science and Technology. - 2013. - V. 68. - P. 577-594. уменьшению размерности пространства поиска. Таким образом, предложенный подход помогает на практике сократить временные затраты на адаптацию модели, столь трудоемкий и важный этап моделирования разработки нефтяных месторождений.

15. New approach to automatic history matching using kernel PCA / P. Sarma, L.J. Durlofsky, K. Aziz, W.H.A. Chen // SPE Reservoir Simulation Symposium. - Houston, USA, 26-28 February, 2007. URL: https://onepetro.org/spersc/proceedings-abstract/07RSS/All07RSS/SPE-106176-MS/143615 (дата обращения: 05.04.2021).

16. Design of objective function for interference well testing / R. Booth, A.C. Bertolini, K.L. Morton, A.J. Fitzpatrick // OTC Brasil. - Rio de Janeiro, Brazil, October, 2013. URL: https://onepetro.org/OTCBRASIL/proceedings-

abstract/13OTCB/All-13OTCB/OTC-24513-MS/40969 (дата обращения: 05.04.2021).

17. Bouzarkouna Z., Nobakht B., Karim N. A better formulation of objective functions for history matching using hausdorff distances // EUROPEC 2015. - Madrid, Spain, 1-4 June, 2015. URL: https://onepetro.org/SPEEURO/proceedings-

abstract/15EURO/All-15EURO/SPE-174302-MS/183479 (дата обращения: 05.04.2021).

18. How does the definition of the objective function influence the outcome of history matching? / G. Eremyan, I. Matveev, G. Shishaev, V. Rukavishnikov, V. Demyanov // ECMOR XVII: Conference Proceedings. - Houten, 14-17 September, 2020. Houten: European Association of Geoscientists \& Engineers, 2020. - V. 2020. - P. 1-14.

19. Ferraro P., Verga F. Use of evolutionary algorithms in single and multi-objective optimization techniques for assisted history matching // Offshore Mediterranean Conference and Exhibition. Ravenna, Italy, 25-27 March, 2009. URL: https://onepetro.org/ OMCONF/proceedings-abstract/OMC09/All-OMC09/OMC-2009079/1101 (дата обращения: 05.04.2021).

20. Park H.Y. Handling conflicting multiple objectives using paretobased evolutionary algorithm for history matching of reservoir performance // SPE Reservoir Simulation Symposium. Woodlands, USA, 18-20 February, 2013. URL: https://onepetro.org/spersc/ proceedings-abstract/13RSS/All13RSS/SPE-163623-MS/177620 (дата обращения: 05.04.2021).

21. Stephen K.D. Seismic history matching with saturation indicators combined with multiple objective function optimization // EAGE Annual Conference \& Exhibition incorporating SPE Europec. London, UK, 10-13 June, 2013. URL: https://onepetro.org/ SPEEURO/proceedings-abstract/13EURO/All-13EURO/SPE 164857-MS/177300 (дата обрашения: 05.04.2021).

22. Еремян Г.А., Рукавишников В.С. Критерии качества автоматизированной адаптации геолого-гидродинамической модели месторождения углеводородов // Экспозиция Нефть Газ. 2020. - № 6. - С. 76-79.

23. Еремян Г.А., Рукавишников В.С. Влияние способов нормировки целевой функции на результаты автоадаптации численной модели месторождения углеводородов // Экспозиция Нефть Газ. - 2020. - № 6. - С. 81-86.

24. Прогнозирование геолого-физических параметров месторождений углеводородов в условиях неопределенности данных / А.И. Кобрунов, В.Е. Кулешов, А.С. Могутов, А.Н. Дорогобед // Нефтяное хозяйство. - 2014. - № 7. - С. 78-80.

25. Reservoir model uncertainty quantification through computerassisted history matching / C. Yang, L.X. Nghiem, C. Card, M. Bremeier // SPE Annual Technical Conference and Exhibition. - Anaheim, USA, 11-14 November, 2007. URL: https://onepetro.org/SPEATCE/proceedings-abstract/07ATCE/All07ATCE/SPE-109825-MS/142893 (дата обращения 05.04.2021)

26. Wilson A. Uncertainty quantification for history-matching problems // Journal of Petroleum Technology. - 2017. - V. 69. - P. 90-92.

27. Hierarchical benchmark case study for history matching, uncertainty quantification and reservoir characterization / D. Arnold, V. Demyanov, D. Tatum, M. Christie, T. Rojas, S. Geiger, P. Corbett // Computers \& Geosciences. - 2013. V. 50. - P. 4-15. 
28. Christie M., Demyanov V., Erbas D. Uncertainty quantification for porous media flows // Journal of Computational Physics. - 2006. V. $127 .-$ P. $143-158$

29. Multi-data reservoir history matching for enhanced reservoir forecasting and uncertainty quantification / K. Katterbauer, S Arango, S. Sun, I. Hoteit // Journal of Petroleum Science and Engineering. - 2015. - V. 128. - P. 160-176.

30. Dake L.P. Fundamentals of reservoir engineering. - Amsterdam: Elsevier, 1978. - 443 p.

31. Reservoir history matching by ensemble smoother with principle component and sensitivity analysis for heterogeneous formations / Q. Liao, K. Alsamadony, G. Lei, A. Awotunde, S. Patil // Journal of Petroleum Science and Engineering. - 2021. - V. 198. - 108140

32. A random forests-based sensitivity analysis framework for assisted history matching / A. Aulia, D. Jeong, I.M. Saaid, D. Kania, M.T. Shuker, N.A. El-Khatib // Journal of Petroleum Science and Engineering. -2019 . - V. 181. -106237.

33. Sensitivity and history match analysis of a carbon dioxide «huffand-puff» injection test in a horizontal shale gas well in Tennessee / C. Keles, X. Tang, C. Schlosser, A.K. Louk, N.S. Ripepi // Journal of Natural Gas Science and Engineering. - 2020. - V. 77. 103226.

34. Kang B., Choe J. Initial model selection for efficient history matching of channel reservoirs using Ensemble Smoother // Journal of Petroleum Science and Engineering. - 2017. - V. 152. P. 294-308.

35. Submarine fan reservoir architecture and heterogeneity influence on hard-to-recover reserves. Achimov Fm / A. Kondratyev, V. Rukavishnikov, L. Shakirzyanov, K. Maksyutin // IOP Conference Series: Earth and Environmental Science. Scientific and Technical Challenges in the Well Drilling Progress. - 2015. V. 24. -012041 .

\section{Информация об авторах}

Eремян Г.А., инженер лаборатории геологии месторождений нефти и газа Национального исследовательского Томского политехнического университета.

Давуди Ш., инженер-исследователь Центра подготовки и переподготовки специалистов нефтегазового дела Национального исследовательского Томского политехнического университета.

Рукавишников B.C., PhD, директор Центра подготовки и переподготовки специалистов нефтегазового дела Национального исследовательского Томского политехнического университета.

Cmenuко A.B., инженер лаборатории геологии месторождений нефти и газа Национального исследовательского Томского политехнического университета.
36. Givens B., Hoffman B.T. A Sensitivity analysis of input parameters for modeling primary and tertiary recovery in unconventional oil reservoirs // SPE Western North American and Rocky Mountain Joint Meeting. - Denver, USA, 17-18 April, 2014. URL: https://onepetro.org/SPEWRM/proceedings(дата 7. Peskova D.N, Sizykh A.V., Rukavishnikov V.S. Evaluation the value-of-Information (VOI) and look back analysis during modelling of the exploration works $/ / 7^{\text {th }}$ EAGE Saint Petersburg International Conference and Exhibition: Conference Proceedings. - St-Petersburg, Russia, April, 2016. - Houten: European Association of Geoscientists \& Engineers, 2016. -

38. Ritzi R.W., Bobeck P. Comprehensive principles of quantitative hydrogeology established by Darcy (1856) and Dupuit (1857) // Water Resources Research. - 2008. - V. 44. - P. 1-14.

39. Bertolini A.C., Schiozer J.D. Influence of the objective function in the history matching process // Journal of Petroleum Science and Engineering. -2011 . - V. 78. - P. 32-41.

40. Oliveira G.S., Schiozer D.J., Maschio C. History matching by integrating regional multi-property image perturbation methods with a multivariate sensitivity analysis // Journal of Petroleum Science and Engineering. - 2017. - V. 153. - P. 111-122.

41. Еремян Г.А. Методика выбора оптимальной целевой функции для автоадаптации геолого-гидродинамической модели // Геология, геофизика и разработка нефтяных и газовых месторождений. - 2021. - № 1. - С. 30-38.

Поступила 19.04.2021 г. P. $480-487$. 
UDC 622.32:519.8

\title{
COMPREHENSIVE APPROACH TO PARAMETRIZATION OF A PETROLEUM RESERVOIR MODEL FOR AUTOMATED HISTORY MATCHING
}

\author{
Grachik A. Eremyan ${ }^{1}$, \\ eremyanga@hw.tpu.ru

\section{Shadfar Davoodi ${ }^{1}$,} \\ davoodis@hw.tpu.ru
}

Valeriy S. Rukavishnikov', rukavishnikovvs@hw.tpu.ru

\author{
Artem V. Stepiko', \\ stepikoav@hw.tpu.ru \\ 1 National Research Tomsk Polytechnic University, \\ 30, Lenin avenue, Tomsk, 634050, Russia.
}

The relevance of the research is caused by the need to select from a variety of parameters of a petroleum reservoir model the most important ones. By adjusting these parameters, the optimization algorithm should find solutions that ensure the correct reproduction of historical data by the model. History matching is a time-consuming and inevitable step in numerical modeling of hydrocarbon reservoirs, which confirms the suitability of the model for predictions. Limiting the number of tuning parameters to the most significant allows the model to be successfully history matched in practice within a reasonable time frame.

The main aim of the work is to determine a set of significant history matching parameters based on the study of the degree and nature of their influence on the convergence of key performance indicators of the wells and the field.

Object: automated history matching of a petroleum reservoir model to the data of a reference case, which is an analogue of historical indicators of real field development.

Methods: numerical geological modelling and reservoir simulation, computational experiment, sensitivity analysis.

Results. The authors have developed the classification of history matching parameters according to the degree of influence and the degree of uncertainty based on the law of fluid flow in a porous medium. Based on multiple computational experiments on the synthetic reservoir model built during the work we studied the degree and nature of the influence of various history matching parameters on the convergence of field development indicators. The use of two types of sensitivity analysis made it possible to characterize both the individual influence of the parameters and the combined influence taking into account the variation of the remaining parameters. Understanding the mutual influence of the parameters is important, since during history matching all tuning parameters are varied simultaneously. The conclusions of the numerical experiments are consistent with the analytical approach. The implemented comprehensive approach makes it possible to form a reasonable set of tuning parameters of the model, ranked according to the degree of importance for history matching.

\section{Key words:}

Sensitivity analysis, model parametrization, history matching parameters, uncertainties,

automated history matching, optimization algorithm, objective function, reservoir simulation.

\section{REFERENCES}

1. Tarantola A. Inverse problem theory and methods for model parameter estimation. Paris, Society for Industrial and Applied Mathematics Publ., 2005. 342 p.

2. Gladkov E.A., Gladkova E.E. Trekhmernaya geologotekhnologicheskaya model mestorozhdeniya UV na osnove individualnoy poskvazhinnoy adaptatsii [Three-dimensional geological and engineering model of hydrocarbon field based on individual well level history matching]. Gazovaya promyshlennost, 2010, no. 5 , pp. $104-108$.

3. Shishaev G.Y., Matveev I.V., Eremyan G.A., Demyanov V.V., Kaygorodov S.V. Geologically plausible computer-aided history matching on the example of one the oil fields. Oil Industry, 2020, no. 6, pp. 58-61. In Rus.

4. Matveev I., Shishaev G., Eremyan G., Demyanov V., Popova O., Kaygorodov S., Belozerov B., Uzhegova J., Konoshonkin D., Korovin M. Geology driven history matching. SPE Russian Petroleum Technology Conference. Moscow, 22-24 October, 2019. Available at: https://onepetro.org/SPERPTC/proceedings-abstract/ 19RPTC/1-19RPTC/D013S001R004/219147 (accessed: 05 April 2021).

5. Matveev I., Shishaev G., Eremyan G., Konoshonkin D., Demyanov V., Kaygorodov S. Geology realism control in automated history matching. ECMOR XVII: Conference Proceedings. Houten, 14-17 September, 2020. Houten, European Association of Geoscientists \& Engineers Publ., 2020, vol. 2020, pp. 1-9.
6. Verga F., Cancelliere M., Viberti D. Improved application of assisted history matching techniques. Journal of Petroleum Science and Engineering, 2013, vol. 109, pp. 327-347.

7. Foroud T., Seifi A., Amin Shahidi B. Assisted history matching using artificial neural network based global optimization method applications to Brugge field and a fractured Iranian reservoir. Journal of Petroleum Science and Engineering, 2014, vol. 123, pp. 46-61.

8. Chai Z., Nwachukwu A., Zagayevskiy Y., Amini S., Madasu S. An integrated closed-loop solution to assisted history matching and field optimization with machine learning techniques. Journal of Petroleum Science and Engineering, 2021, vol. 198, no. 108204.

9. Xavier C.R., Santos E.P., Vieira V.F., Santos R.W. Genetic algorithm for the history matching problem. Procedia Computer Science, 2013. vol.18, pp. 946-955.

10. Rwechungura R.W., Dadashpour M., Kleppe J. Advanced history matching techniques reviewed. SPE Middle East Oil and Gas Show and Conference. Manama, Bahrain, September, 2011. Available at: https://onepetro.org/SPEMEOS/proceedings-abstract/ 11MEOS/All-11MEOS/SPE-142497-MS/148210 (accessed: 05 April 2021).

11. Almeida F.L.R., Davolio A., Schiozer D.J. A New approach to perform a probabilistic and multi-objective history matching. SPE Annual Technical Conference and Exhibition. Amsterdam, Netherlands, October, 2014. Available at: https://onepetro.org/ SPEATCE/proceedings-abstract/14ATCE/All-14ATCE/SPE170623-MS/211614 (accessed: 05 April 2021). 
12. Al-Sofi A.M., Blunt M.J. Control of numerical dispersion in simulations of augmented waterflooding. SPE Improved Oil Recovery Symposium. Tulsa, USA, April 2010. Available at: https://onepetro.org/SPEIOR/proceedings-abstract/10IOR/All10IOR/SPE-129658-MS/107593 (accessed: 05 April 2021).

13. Gervais-Couplet V., Roggero F., Feraille M., Le Ravalec-Dupin M., Seiler A. Joint history matching of production and 4d-seismic related data for a north sea field case. SPE Annual Technical Conference and Exhibition. Florence, Italy, September, 2010. Available at https://onepetro.org/SPEATCE/proceedings-abstract/10ATCE/ All10ATCE/SPE-135116-MS/102106 (accessed: 05 April 2021).

14. Kazemi A., Stephen K.D. Optimal parameter updating in assisted history matching using streamlines as a guide. Oil \& Gas Science and Technology, 2013, vol. 68, pp. 577-594.

15. Sarma P., Durlofsky L.J., Aziz K., Chen W.H. A new approach to automatic history matching using kernel PCA. SPE Reservoir Simulation Symposium. Houston, USA, 26-28 February, 2007. Available at: https://onepetro.org/spersc/proceedings-abstract/07RSS/All-07RSS/ SPE-106176-MS/143615 (accessed: 05 April 2021).

16. Booth R., Bertolini A.C., Morton K.L., Fitzpatrick A.J. Design of objective function for interference well testing. OTC Brasil. Rio de Janeiro, Brazil, October, 2013. Available at: https://onepetro. org/OTCBRASIL/proceedings-abstract/13OTCB/All13OTCB/OTC-24513-MS/40969 (accessed: 05 April 2021)

17. Bouzarkouna Z., Nobakht B., Karim N. A better formulation of objective functions for history matching using hausdorff distances. EUROPEC 2015. Madrid, Spain, 1-4 June, 2015. Available at: https://onepetro.org/SPEEURO/proceedingsabstract/15EURO/All-15EURO/SPE-174302-MS/183479 cessed: 05 April 2021).

18. Eremyan G., Matveev I., Shishaev G., Rukavishnikov V., Demyanov V. How does the definition of the objective function influence the outcome of history matching? ECMOR XVII: Conference Proceedings. Houten, 14-17 September, 2020. Houten, European Association of Geoscientists \& Engineers Publ., 2020. Vol. 2020, pp. 1-14.

19. Ferraro P., Verga F. Use of evolutionary algorithms in single and multi-objective optimization techniques for assisted history matching. Offshore Mediterranean Conference and Exhibition. Ravenna, Italy, 25-27 March, 2009. Available at: https://onepetro.org/ OMCONF/proceedings-abstract/OMC09/All-OMC09/OMC-2009079/1101 (accessed: 05 April 2021).

20. Park H.Y. Handling conflicting multiple objectives using paretobased evolutionary algorithm for history matching of reservoir performance. SPE Reservoir Simulation Symposium. Woodlands, USA, 18-20 February, 2013. Available at: https://onepetro.org/ spersc/proceedings-abstract/13RSS/All-13RSS/SPE-163623MS/177620 (accessed: 05 April 2021).

21. Stephen K.D. Seismic history matching with saturation indicators combined with multiple objective function optimization. $E A G E$ Annual Conference \& Exhibition incorporating SPE Europec. London, UK, 10-13 June, 2013. Available at: https://onepetro. org/SPEEURO/proceedings-abstract/13EURO/All-13EURO/SPE164857-MS/177300 (accessed: 05 April 2021).

22. Eremyan G.A., Rukavishnikov V.S. Quality criteria for automated history matching of hydrocarbon reservoir models. Exposition Oil Gas, 2020, no. 6, pp. 76-79. In Rus.

23. Eremyan G.A., Rukavishnikov V.S. Influence of the objective function normalization method on the reservoir model history matching results. Exposition Oil Gas, 2020, no. 6, pp. 81-86. In Rus

24. Kobrunov A.I., Kuleshov V.E., Mogutov A.S., Dorogobed A.N. Prediction of hydrocarbon deposit physical and geological commercial parameters under conditions of data uncertainty. Oil Industry, 2014, no. 7, pp. 78-80. In Rus.

25. Yang C., Nghiem L.X., Card C., Bremeier M. Reservoir model uncertainty quantification through computer-assisted history matching. SPE Annual Technical Conference and Exhibition. Anaheim, USA, 11-14 November, 2007. Available at:
https://onepetro.org/SPEATCE/proceedings-abstract/07ATCE/All07ATCE/SPE-109825-MS/142893 (accessed: 05 April 2021).

26. Wilson A. Uncertainty quantification for history-matching problems. Journal of Petroleum Technology, 2017, vol. 69, pp. 90-92.

27. Arnold D., Demyanov V., Tatum D., Christie M., Rojas T., Geiger S., Corbett P. Hierarchical benchmark case study for history matching, uncertainty quantification and reservoir characterization. Computers \& Geosciences, 2013, vol. 50, pp. 4-15.

28. Christie M., Demyanov V., Erbas D. Uncertainty quantification for porous media flows. Journal of Computational Physics, 2006, vol. 127. pp. 143-158

Katterbauer K., Arango S., Sun S., Hoteit I. Multi-data reservoir history matching for enhanced reservoir forecasting and uncertainty quantification. Journal of Petroleum Science and Engineering, 2015, vol. 128, pp. 160-176.

29. Dake L.P. Fundamentals of reservoir engineering. Amsterdam, Elsevier Publ., 1978. 443 p

30. Liao Q., Alsamadony K., Lei G., Awotunde A., Patil S. Reservoir history matching by ensemble smoother with principle component and sensitivity analysis for heterogeneous formations. Journal of Petroleum Science and Engineering, 2021, vol. 198, no. 108140.

31. Aulia A., Jeong D., Saaid I.M., Kania D., Shuker M.T., El-Khatib N.A. A random forests-based sensitivity analysis framework for assisted history matching. Journal of Petroleum Science and Engineering, 2019, vol. 181, no. 106237

32. Keles C., Tang X., Schlosser C., Louk A.K., Ripepi N.S. Sensitivity and history match analysis of a carbon dioxide «huff-and-puff» injection test in a horizontal shale gas well in Tennessee. Journal of Natural Gas Science and Engineering, 2020, vol. 77, no. 103226.

33. Kang B., Choe J. Initial model selection for efficient history matching of channel reservoirs using Ensemble Smoother. Journal of Petroleum Science and Engineering, 2017, vol. 152, pp. 294-308.

34. Kondratyev A., Rukavishnikov V., Shakirzyanov L., Maksyutin K. Submarine fan reservoir architecture and heterogeneity influence on hard-to-recover reserves. Achimov Fm. IOP Conference Series: Earth and Environmental Science. Scientific and Technical Challenges in the Well Drilling Progress, 2015, vol. 24, no. 012041.

35. Givens B., Hoffman B.T. A sensitivity analysis of input parameters for modeling primary and tertiary recovery in unconventional oil reservoirs. SPE Western North American and Rocky Mountain Joint Meeting. Denver, USA, 17-18 April, 2014. Available at: https://onepetro.org/SPEWRM/proceedings-

abstract/14WRRM/All-14WRRM/SPE-169530-MS/211285 (accessed: 05 April 2021)

36. Peskova D.N., Sizykh A.V., Rukavishnikov V.S. Evaluation the value-of-Information (VOI) and look back analysis during modelling of the exploration works. $7^{\text {th }}$ EAGE Saint Petersburg International Conference and Exhibition. St-Petersburg, April, 2016. Houten, European Association of Geoscientists \& Engineers Publ., 2016. pp. $480-487$.

37. Ritzi R.W., Bobeck P. Comprehensive principles of quantitative hydrogeology established by Darcy (1856) and Dupuit (1857). Water Resources Research, 2008, vol. 44, pp. 1-14.

38. Bertolini A.C., Schiozer J.D. Influence of the objective function in the history matching process. Journal of Petroleum Science and Engineering, 2011, vol. 78, pp. 32-41.

39. Oliveira G.S., Schiozer D.J., Maschio C. History matching by integrating regional multi-property image perturbation methods with a multivariate sensitivity analysis. Journal of Petroleum Science and Engineering, 2017, vol. 153, pp. 111-122.

40. Eremyan G.A. Methodology for selecting the optimal objective function for reservoir model history. Geology, Geophysics and Development of Oil and Gas Fields, 2021, no. 1, pp. 30-38. In Rus.

Received: 19 April 2021.

Information about the authors

Grachik A. Eremyan, engineer, National Research Tomsk Polytechnic University.

Shadfar Davoodi, research engineer, National Research Tomsk Polytechnic University.

Valeriy S. Rukavishnikov, PhD, director of Petroleum Learning Centre, National Research Tomsk Polytechnic University. Artem V. Stepiko, engineer, National Research Tomsk Polytechnic University. 